\title{
Editorial: Non-target Effects of Pesticides on Organisms Inhabiting Agroecosystems
}

\author{
Johann G. Zaller ${ }^{1 *}$ and Carsten A. Brühl ${ }^{2}$ \\ ${ }^{1}$ Department of Integrative Biology and Biodiversity Research, Institute of Zoology, University of Natural Resources and Life \\ Sciences (BOKU), Vienna, Austria, ${ }^{2}$ Community Ecology and Ecotoxicology, Institute for Environmental Sciences, University \\ of Koblenz-Landau, Landau, Germany
}

Keywords: ecotoxicology, plant protection, agroecology, regulatory toxicity, agrochemicals

\section{Editorial on the Research Topic}

Non-target Effects of Pesticides on Organisms Inhabiting Agroecosystems

\section{OPEN ACCESS}

Edited and reviewed by: Peter Thorburn,

Commonwealth Scientific and Industrial Research Organisation (CSIRO), Australia

*Correspondence: Johann G. Zaller johann.zaller@boku.ac.at

Specialty section:

This article was submitted to Agroecology,

a section of the journal

Frontiers in Environmental Science

Received: 23 January 2019

Accepted: 14 May 2019

Published: 31 May 2019

Citation:

Zaller JG and Brühl CA (2019) Editorial: Non-target Effects of Pesticides on Organisms Inhabiting Agroecosystems.

Front. Environ. Sci. 7:75. doi: 10.3389/fenvs.2019.00075
Pesticides are increasingly used worldwide and concurrently, evidence is mounting that they have detrimental ecological effects on the biodiversity of organisms in agricultural landscapes. However, pesticides are also perceived as a highly regulated group of chemicals in respect of their risk for the environment. The aim of this Research Topic, is to provide an overview of the state of knowledge regarding non-target effects of pesticides and to identify knowledge gaps. We collated in total 14 papers written by 61 authors, eight of which are original research papers and six are reviews. Many papers dealt with effects of herbicides and insecticides. Effects of glyphosate and neonicotinoids were addressed for various groups of organisms. But also fungicides as well as rodenticides were in the focus of researchers and some addressed more than one pesticide class. Several papers explicitly made recommendations for amendments to current risk assessment approaches required for the regulation of pesticides.

The mode-of-action of glyphosate-based herbicides is still not completely understood. Gomes et al. tested glyphosate-dependent inhibition of photosynthesis in willow. The authors showed that glyphosate promotes changes in the photosynthetic apparatus leading to decreased photochemistry. For the first time, interconnecting effects on shikimate pathway, photosynthetic process and oxidative events in plants were presented. This research documented that glyphosate induces a series of interconnected events that leads to decreased photosynthetic activity in willow plants and indicates how glyphosate tolerance in plants may develop through the activation of antioxidant systems.

Herbicide drift effects in field margin plant community have been little studied so far. In a field trial Nelemans et al. investigated effects of an herbicide (metsulfuron-methyl) on sown plant communities. The herbicide drift affected biomass production, plant cover and seed germination of several plant species. The study implies that spray drift leads to shifts in species compositions and succession in vegetation in off-crop areas adjacent to arable fields.

Herbicides can not only affect the off-field plants but also soil microorganisms as studied by Aristilde et al. Their results show that glyphosate altered metabolite levels in the biosynthetic pathway of aromatic amino acids in the soil bacterium Pseudomonas. The greatest inhibition was found for tryptophan, an important precursor to secondary metabolites. The authors conclude that the glyphosate-induced specific disruption of de novo biosynthesis of aromatic amino acids accompanied by widespread metabolic disruptions was responsible for the observed dosedependent adverse effects. Their findings suggest that rhizospheric bacteria may be less susceptible to glyphosate effects due to the high-carbon environment in the rhizosphere with relatively higher concentration of amino acids and sugars relative to bulk soils. 
It is rarely considered that amphibian species migrating through arable fields may be exposed to pesticides. Berger et al. evaluated regional migration patterns and glyphosate applications in German agriculture over 20 years. Their results reveal the highest increase in temporal coincidence with glyphosate application for both adult and juvenile Great crested newt (Triturus cristatus) and Fire-bellied toad (Bombina bombina). The authors demand that pesticide risk assessment should start considering potential amphibian exposure and include resulting effects in the regulatory procedure.

Interactive effects between glyphosate-based herbicides and environmental parameters were studied by Baier et al. Results of this laboratory experiment showed that eggs of Common toads (Bufo bufo) were more sensitive to herbicides than tadpoles. Interaction between herbicide concentrations and temperature resulted in more pronounced glyphosate effects at lower temperatures. This is remarkable as ecotoxicologial studies for regulatory risk assessment are not evaluating temperature as a co-stressor and therefore the actual risk might be underestimated.

In their contribution, Gomes and Juneau reviewed how variations in climatic conditions influence herbicide toxicity in algae and cyanobacteria. They show that responses to interactions between light, temperature, and herbicides are species-specific, making it difficult to establish a single model of how climate change will affect herbicide toxicity for aquatic algae. The authors also demand the inclusion of environmental parameters when assessing pesticide risk.

In his mini-review Stoner shows that current neonicotinoid risk assessment does not adequately address differences between honey bees (Apis mellifera) and bumble bees (Bombus spp.). Because bumble bee queens are solitary for some time and forage for pollen and nectar themselves, their pesticide exposure is higher than for honey bee queens, where worker bees provide food and are therefore potentially exposed to pesticides. Additional research focusing on critical periods in a bumble bee queen's life and the risk for pesticide exposure and related effects is demanded.

Neonicotinoids and their effects on butterflies, including also sublethal endpoints, were addressed in a systematic review by Mulé et al. The insecticides cause negative effects such as reduced survival rate, feeding interruption, and alteration of oviposition behavior. The paper highlights that it has been impossible to determine which butterfly species is the most sensitive so far.

The impact of insecticides on aquatic environments was reviewed by Sánchez-Bayo et al. The paper shows a widespread aquatic contamination by neonicotinoids and identifies the communities most at risk. Gaps in knowledge stem from difficulties in obtaining long-term experimental data relating effects on individual organisms to impacts on populations and ecosystems.

Single and combined effects of insecticide and fungicide seed dressings and subsequent herbicide application on soil biota and processes were studied by van Hoesel et al. Seed dressings in winter wheat significantly reduced the surface activity of earthworms with no difference whether insecticides or fungicides were used. The authors conclude that interactive effects on soil biota and processes of different systemic pesticide classes should receive more attention.

Although agriculture dominates much of Europe's landscape, there is no information on bat activity in different crops and therefore exposure cannot be addressed appropriately. Stahlschmidt et al. investigated foraging activity of bats in an agricultural landscape in Germany. In 300 accumulated sampling nights a total of 14 bat species were recorded. The high bat activity levels above crops, related pesticide inputs and the availability of prey insects, makes a dietary exposure of bats likely. Bats and their exposure toward pesticides are currently not considered in the EU pesticide regulation.

An often-ignored aspect was addressed by Topping and Elmeros: The exposure of mammalian predators to anticoagulant rodenticides was investigated using a spatio-temporal model supported by an experimental study. The paper suggests that the driver of high anticoagulant rodenticides incidence in non-target small mammal predators is likely to be related to use patterns.

In their review Römbke et al. summarized ecotoxicological effects of pesticides on enchytraeids (Oligochaeta) in agroecosystems. Because of their close contact with the soil pore water, a high ingestion rate and a thin cuticle, they show a high sensitivity to a broad range of pesticides. The authors recommend the use of enchytraeids in pesticide risk assessments because of their diversity, functional importance and simple use in standardized tests.

In their review, Ittner et al. draw attention to aquatic fungi as a disregarded trophic level, not addressed in regulatory aquatic risk assessment so far. Freshwater fungi are a diverse group and fulfill important functions in the food web dynamics of surface water ecosystems. The authors conclude that development and standardization of different fungi bioassays is needed to effectively protect food-webs in aquatic ecosystems.

Taken collectively, we are grateful to all contributors for presenting such a variety of aspects at different complexity levels from physiology to ecosystem functioning. It also became clear that we deploy enormous amounts of pesticides that reach different compartments of the ecosystems in the agricultural landscape with little knowledge on their non-target effects. As a consequence, dramatic declines in biodiversity in insects, birds and other organisms have been related to pesticide pollution (Geiger et al., 2010; Beketov et al., 2013; Sánchez-Bayo and Wyckhuys, 2019). This should alert us to radically challenge and completely remodel the current procedures for environmental risk assessments of pesticides as they are obviously inadequate to protect the biodiversity and integrity of ecosystems. All contributions from this Research Topic also highlighted a great array of remaining knowledge gaps, among them are (not exhaustive):

- effects of additives and surfactants in pesticide formulations rather than active ingredients

- effects on realistic field populations and communities rather than singe species studies

- effects of more than one pesticides resulting from tank mixtures and real-life application sequences

- carry-over and legacy effects 
- effects in agricultural fields and neighboring ecosystems (e.g., pesticide drift)

- interactions with environmental stressors such as organismic competition, abiotic factors (temperature, moisture), soil types (humus content), and climate change

In conclusion, priorities for future work on pesticides and their effects should focus on investigating or simulating realistic field

\section{REFERENCES}

Beketov, M. A., Kefford, B. J., Schäfer, R. B., and Liess, M. (2013). Pesticides reduce regional biodiversity of stream invertebrates. Proc. Natl. Acad. Sci. U.S.A. 110, 11039-11043. doi: 10.1073/pnas.1305618110

Geiger, F., Bengtsson, J., Berendse, F., Weisser, W. W., Emmerson, M., and Morales, M. B., (2010). Persistent negative effects of pesticides on biodiversity and biological control potential on European farmland. Basic Appl. Ecol. 11, 97-105. doi: 10.1016/j.baae.2009.12.001

Sánchez-Bayo, F., and Wyckhuys, K. A. G. (2019). Worldwide decline of the entomofauna: a review of its drivers. Biol. Conserv. 232, 8-27. doi: 10.1016/j.biocon.2019.01.020 situations, i.e., multiple applications of pesticides during the growing season including their temporal and spatial interactions with fauna and flora.

\section{AUTHOR CONTRIBUTIONS}

All authors listed have made a substantial, direct and intellectual contribution to the work, and approved it for publication.

Conflict of Interest Statement: The authors declare that the research was conducted in the absence of any commercial or financial relationships that could be construed as a potential conflict of interest.

Copyright (c) 2019 Zaller and Brühl. This is an open-access article distributed under the terms of the Creative Commons Attribution License (CC BY). The use, distribution or reproduction in other forums is permitted, provided the original author(s) and the copyright owner(s) are credited and that the original publication in this journal is cited, in accordance with accepted academic practice. No use, distribution or reproduction is permitted which does not comply with these terms. 